\section{Foliar Application of Liquid Urea- triazone-based Nitrogen Fertilizers and Crop Safety}

\author{
J.G. Clapp, Jr.'
}

Additional index words. S-tetrahydrotriazone, slowly available nitrogen, leaf injury, vegetable crops, fruit crops, nut crops, field crops

Summary. Urea-triazone-based nitrogen (N) solutions were evaluated for potential leaf injury on agronomic and horticultural crops at 61 commercial grower sites throughout the United States. Poliar spray solutions containing triazone $\mathbf{N}$ were used at concentrations ranging from $1.5 \%$ to $15.7 \%$. Safe $\mathbf{N}$ concentrations for urea-triazone-based $\mathbf{N}$ products ranged from $1.5 \%$ for crops such as sweet corn, apple, cherry, and pear, and up to $15.7 \%$ for nursery root stocks. Ureatriazone-based $\mathbf{N}$ solutions were found to be much safer on crop foliage than ammonium-, nitrate-, and/ or all urea-based foliar fertilizer products than reported in the literature.

$\mathrm{M}$ ost foliar nitrogen $(\mathrm{N})$ products developed prior to the discovery and commercialization of urea-triazone solutions contained ammonium, nitrate, and/or urea $\mathrm{N}$ sources. These sources have a very high osmolality index, which results in a high burn potential when applied to plant foliage (Clapp and Parham, 1991). Due to this burn potential, products developed from these sources generally are applied at low application rates and in dilute spray concentrations.

Arcadian Corporation, 310 Clapp Furms Road, Greensboro, NC 27305.

'Manager. Agronomics and Commercial Development, Triazone Division.

The cost of publishing this paper was defrayed in part by the payment of page charges. Under postal regulations, this paper therefore must hereby be marked advertisement solely to indicate this fact.
Embleton et al. (1986) reported that, in order to avoid the toxic effects of dilute urea sprays on citrus, the concentration should not exceed $14 \mathrm{~g}$ urea/ liter, or $0.64 \%$ N. Ferrer and Forshey (1988) used only $6 \mathrm{~g}$ urea/liter $(0.28 \%$ $\mathrm{N})$ concentration on apples in Ohio. Ippersiel et al. (1989) selected a $0.88 \%$ $\mathrm{N}$ concentration of urea for applications on corn, and considered it to be the highest practical safe application rate without causing leaf injury.

The potential for leaf injury from foliar fertilizer products containing urea as the $\mathrm{N}$ source, along with other nutrients, has limited the rate of application. For example, Jones (1989) reported that the manufacturer of a $\mathrm{N}$ $\mathrm{P}_{2} \mathrm{O}_{5}-\mathrm{K}_{2} \mathrm{O}$ plus micronutrient product (12-6-6) suggested that the product be applied in a spray concentration of only $0.25 \%$.

Urea-triazone liquid $\mathrm{N}$ fertilizers are produced by the Triazone Division, Arcadian Corp., Memphis, Tenn. These solutions contain a patented,

Table 1. Safe foliar nitrogen concentrations observed on selected crops from a urea-triazonebased $N$ solution (N-Sure: 28-0-0)z.

\begin{tabular}{|c|c|c|c|c|}
\hline \multirow[b]{2}{*}{ Crop } & \multirow{2}{*}{$\begin{array}{c}\text { Growth } \\
\text { stage }\end{array}$} & \multicolumn{2}{|c|}{$\begin{array}{c}\text { Application rate } \\
\text { (gal/acre) }\end{array}$} & \multirow{2}{*}{$\begin{array}{l}\text { Spray } \mathbf{N} \\
\text { concn. (\%) }\end{array}$} \\
\hline & & N-Sure & Water & \\
\hline Cauliflower & Six-leaf & 10 & 40 & 6.8 \\
\hline Cherry (Bing) & Full leaf & 20 & 80 & 6.8 \\
\hline Grape (Thompson Seedless) & Canes 2 to $3 \mathrm{ft}$ & 10 & 40 & 6.8 \\
\hline Lettuce & Heading & 10 & 40 & 6.8 \\
\hline Pepper & 4 inches & 10 & 40 & 6.8 \\
\hline Spinach & Midway & 10 & 40 & 6.8 \\
\hline Tomatoes & Closing row & 20 & 30 & 13.2 \\
\hline $\begin{array}{l}\text { Nursery rootstock } \\
\text { (Almond, Marianna 29C, } \\
\text { Nemagard, Nemared) }\end{array}$ & 30 Apr. & 20 & 20 & 15.7 \\
\hline
\end{tabular}

${ }^{z} N$-Sure applied at rates up to 20 gal/acre (inpublished data from D.T. Schulteis, Fresno, Calif.)

Table 2. Safe foliar nitrogen concentrations on vegetable crops from a urea-triazone-based nutrient solution (Trisert: 13-3-4).

\begin{tabular}{|c|c|c|c|c|c|}
\hline \multirow[b]{2}{*}{ Crop } & \multirow[b]{2}{*}{ Growth stage } & \multirow[b]{2}{*}{ Location } & \multicolumn{2}{|c|}{$\begin{array}{c}\text { Application rate } \\
\text { (gal/acre) }\end{array}$} & \multirow{2}{*}{$\begin{array}{l}\text { Spray N } \\
\text { concn. }(\%\end{array}$} \\
\hline & & & Trisert & Water & \\
\hline Asparagus & Fern & Mich. & 20 & 0 & 13.I \\
\hline Beans (green) & First trifoliate & Mich. & 10 & 10 & 7.1 \\
\hline Beans (pinto) & Midpod & N.D. & 5 & 15 & 3.7 \\
\hline Broccoli & Before button & Calif. & 15 & 5 & 10.2 \\
\hline Cabbage & Early head & Texas & 15 & 5 & 10.2 \\
\hline Cauliflower & 10-leaf & Mich. & 20 & 0 & 13.1 \\
\hline Collards & Midharvest & Texas & 20 & 0 & 13.1 \\
\hline Corn (sweet) & Four- to five-leaf & Calif. & 5 & 45 & 1.5 \\
\hline Corn (sweet) & Five-leaf & Mich. & 5 & 15 & 3.7 \\
\hline Cucumber & Three- to four-leaf & Mich. & 20 & 0 & 13.1 \\
\hline Cucumber & Early bloom & Texas & 15 & 5 & 10.2 \\
\hline Dill & Early harvest & Texas & 20 & 0 & 13.1 \\
\hline Kale & Midharvest & Texas & 20 & 0 & 13.1 \\
\hline Lettuce & Four- to five-leaf & Calif. & 5 & 15 & 3.7 \\
\hline Lettuce & Capping & Calif. & 5 & 15 & 3.7 \\
\hline Pepper (bell) & 5 to 6 inches & Texas & 20 & 0 & 13.1 \\
\hline Pepper (bell) & 6 inches & Calif. & 20 & 30 & 5.8 \\
\hline Pepper (bell) & After first picking & Calif. & 10 & 40 & 3.0 \\
\hline Potato & 14 days after emergence & Calif. & 20 & 30 & 5.8 \\
\hline Potato & Late tuber & Calif. & 10 & 10 & 7.1 \\
\hline Squash (zucchini) & Five-leaf & Mich. & 20 & 0 & 13.1 \\
\hline Squash & Midharvest & Texas & 15 & 5 & 10.2 \\
\hline Tomato & 5 to 6 inches & Calif. & 20 & 30 & 5.8 \\
\hline Tomato (processing) & Full bloom & Calif. & 20 & 30 & 5.8 \\
\hline
\end{tabular}


Table 3. Safe foliar nitrogen concentrations obserped on berries, fruits, nuts, vines, and Christmas trees from a urea-triazone-based nutrient solution (Trisert: 13-3-4). ${ }^{z}$

\begin{tabular}{|c|c|c|c|c|c|}
\hline \multirow[b]{2}{*}{ Crop } & \multirow[b]{2}{*}{ Growth stage } & \multirow[b]{2}{*}{ Location } & \multicolumn{2}{|c|}{$\begin{array}{l}\text { Application rate } \\
\text { (gal/acre) }\end{array}$} & \multirow{2}{*}{$\begin{array}{c}\text { Spray N } \\
\text { concn. (\%) }\end{array}$} \\
\hline & & & Trisert & Water & \\
\hline Almond & Full leaf & Calif. & 20 & 30 & 5.8 \\
\hline Apple & 0.5 -inch fruit & Mich. & 15 & 5 & 10.2 \\
\hline Apple & Full bloom & N.Y. & 10 & 10 & 1.5 \\
\hline Blueberry & Early fruit coloring & Mich. & 10 & 10 & 7.1 \\
\hline Muskmelon & 1-inch fruit & Texas & 15 & 5 & 10.2 \\
\hline Cherry (sour) & Petal fall & N.Y. & 10 & 40 & 3.0 \\
\hline Cherry (sour) & Mature foliage & N.Y. & 5 & 45 & 1.5 \\
\hline $\begin{array}{r}\text { Christmas tree } \\
\text { (Douglas fir) }\end{array}$ & Budbreak & Mich. & 20 & 0 & 13.1 \\
\hline $\begin{array}{l}\text { Grape (French } \\
\text { colombard) }\end{array}$ & Full leaf & Calif. & 20 & 30 & 5.8 \\
\hline Orange & 0.25 -inch fruit & Texas & 20 & 0 & 13.1 \\
\hline Peach & Full leaf & Calif. & 20 & 30 & 5.8 \\
\hline Peach & 0.75 -inch fruit & Mich. & 20 & 0 & 13.1 \\
\hline Pear & Late bloom & N.Y. & 5 & 45 & 1.5 \\
\hline Plum & Full leaf & Calif. & 20 & 30 & 5.8 \\
\hline Plum & 0.5 -inch fruit & Mich. & 10 & 10 & 7.1 \\
\hline Strawberry & Prebloom & N.Y. & 10 & 10 & 7.1 \\
\hline Strawberry & Bloom & Mich. & 20 & 0 & 13.1 \\
\hline Watermelon & Early bloom & Texas & 20 & 0 & 13.1 \\
\hline
\end{tabular}

${ }^{\Sigma}$ Trisert solution applied at rates up to 20 gal/acre in a spray polume of either 20 or 50 gal/acre.

Table 4. Safe foliar nitrogen concentrations observed on field crops from a urea-triazone-based nutrient solution (Trisert: 13-3-4).

\begin{tabular}{|c|c|c|c|c|c|}
\hline \multirow[b]{2}{*}{ Crop } & \multirow[b]{2}{*}{ Growth stage } & \multirow[b]{2}{*}{ Location } & \multicolumn{2}{|c|}{$\begin{array}{c}\text { Application rate } \\
\text { (gal/acre) }\end{array}$} & \multirow{2}{*}{$\begin{array}{c}\text { Spray N } \\
\text { concn. (\%) }\end{array}$} \\
\hline & & & Trisert & Water & \\
\hline Flax & First bloom & N.D. & 20 & 0 & 13.1 \\
\hline Flax & Boll development & N.D. & 20 & 0 & 13.1 \\
\hline Oat & Heading & N.C. & 20 & 0 & 13.1 \\
\hline Rice & 0.5 -inch head & Mo. & 20 & 0 & 13.1 \\
\hline Rice & Early head emergence & Mo. & 20 & 0 & 13.1 \\
\hline Soybean & Early bloom & N.C. & 15 & 5 & 10.2 \\
\hline Sugar beet & Seven- to 11-leaf & N.D. & 20 & 0 & 13.1 \\
\hline Sunflower & Early bloom & N.D. & 10 & 10 & 7.1 \\
\hline Sunflower & Early seed fill & N.D. & 10 & 10 & 7.1 \\
\hline Tobacco & Six- to eight-leaf & N.C. & 20 & 0 & 13.1 \\
\hline Wheat & Heading & N.C. & 20 & 0 & 13.1 \\
\hline
\end{tabular}

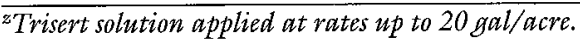

heterocyclic, organic $\mathrm{N}$ compound identified by Hawkins (1985, 1986, $1988)$ as S-tetrahydrotriazone and described by Landels et al. (1990). Urea-triazone solutions have been recognized by the Assn. of American Plant Food Control Officials (AAPFCO, 1991) as a source of slowly available N.

Urea-triazone solutions were evaluated first on turf grasses and were found to be much safer than urea or urea-ammonium nitrate solutions, especially when applied as concentrated solutions (Clapp and Parham, 1991).

The commercial urea-triazone product, "N-Sure" solution, is a clear liquid product that contains $28 \%$ total $\mathrm{N}$. This product contains $3 \mathrm{lb} \mathrm{N} / \mathrm{gal}$ (360 g N/liter) and has a salt-outtemperature (SOT) of <OF (-17.X). Hodgson and MacLeod (1988) were unable to use urea solutions, made by dissolving granular urea on cotton, if the $\mathrm{N}$ concentration was >167 $\mathrm{g} \cdot$ liter $^{-1}$, because of low solubility.

\section{Results}

$\mathrm{N}$-Sure solution, originally developed for use on turf, has been evaluated as a foliar $\mathrm{N}$ source on several crops at $\mathrm{N}$ rates up to $60 \mathrm{lb} / \mathrm{acre}$ (67 $\left.\mathrm{kg} \cdot \mathrm{ha}^{-1}\right)$ and at spray concentrations up to $15.7 \% \mathrm{~N}$. The results from a California cooperator are summarized in Table 1. No leafinjury was observed for these applications on selected crops. The $\mathrm{N}$ concentrations were up to 25 times the rate reported by Embleton et al. (1986) to be acceptable on citrus.

$\mathrm{AN}-\mathrm{P}_{2} \mathrm{O}_{5}-\mathrm{K}_{2} \mathrm{O}$ product containing urea-triazone solution as the $\mathrm{N}$ source, Trisert (13-34) solution, was evaluated extensively for potential leaf injury on a number of crops throughout the United States (Tables 24). Field trials were established, primarily in major vegetable and fruit production regions, to determine the crop safety level for application rates of 5 , 10, 15, and $20 \mathrm{gal} / \mathrm{acre}$ (47, 94, 141, and 188 liter $\cdot$ ha $\left.^{-1}\right)$. At most locations a maximum spray volume of $20 \mathrm{gal} /$ acre was used for vegetable and field crops. The \&gal/acre Trisert solution rate was applied with $15 \mathrm{gal} / \mathrm{acre}$ of water, whereas the 20 -gal/acre rate was applied undiluted.

Trisert solution contains $1.3 \mathrm{lb}$ N/gal (156 g N/liter) and was applied at concentrations of $3.7 \%, 7.1 \%, 10.2 \%$, and $13.1 \% \mathrm{~N}$ for the four rates evaluated. All of these rates are much higher than the $\mathrm{N}$ concentrations being recommended and applied with ammonium, nitrate, and/or all urea-based foliar fertilizer products.

Crops, growth stage, and locations varied considerably, depending on the time of year when the trials were initiated for a given region. Leafinjury observations generally were made 7 to 10 days after treatment. If signs of leaf burn were observed, they were recorded, even if as low as $1 \%$ of the crop foliage showed burns.

Tables 24 summarize the safe concentrations observed for these field trials. A total of 53 crop-growth, stagelocation observations were noted. Of these, 21 were at the maximum undiluted Trisert solution application rate of $20 \mathrm{gal} / \mathrm{acre}$, or $13.1 \% \mathrm{~N}$.

Crops most sensitive to Trisert solution applications were sweet corn, apple, cherry, and pear, but they were still safe at a $\mathrm{N}$ concentration of $1.5 \%$, which is 2 to 5 times higher than reported from all urea $\mathrm{N}$ sprays.

These results illustrate that significantly higher $\mathrm{N}$ rates and/or spray concentrations can be applied safely to crop foliage when the $\mathrm{N}$ source is from a urea-triazone solution. 


\section{TECHNOLOGY AND PRODUCT REPORT}

\section{Literature Cited}

Assn. of American Plant Food Control Officials. 1991. Official fertilizer terms and definitions. Offic. Publ. 44:90-91.

Clapp, J.G., JY., and T.M. Parham, Jr. 1991. Properties and uses of urea-triazonebased nitrogen fertilizers. Fert. Res. 28: 229-232.

Embleton, T.W., M. Matsummura, L.H. Stolzy, D.A. Devitt, W.W. Jones, R. ElMotaium and L.L. Summers. 1986. Citrus nitrogen fertilizer management, groundwater pollution, soil salinity, and nitrogen balance. Applied Agr. Res. 157-64.
Ferree, D.C. and C.G. Forshey. 1988. Influence of pruning and urea sprays on growth and fruiting ofspur-bound 'Delicious' apple trees. J. Amer. Soc. Hort. Sci. 113:699-703.

Hawkins, E.F. 1985. Triazone fertilizer and method of making. U.S. Patent 4,554,005. Issued 19 Nov.

Hawkins, E.F. 1986. Triazone fertilizer and method of making. U.S. Patent 4,599,102. Issued 8 July.

Hawkins, E.F. 1988. Triazone fertilizer and method of making. U.S. Patent 4,778,510. Issued 18 Oct.

Hodgson, AS. and D.A. MacLeod. 1988. Seasonal and soil fertility effects on the response of waterlogged cotton to foliarapplied nitrogen fertilizer. Agron. J. 80:259-265.

Ippersiel, D., I. Alli, A.F. MacKenzie, and G.R. Mehuys. 1989. Nitrogen distribution, yield, and quality of silage corn after foliar nitrogen fertilization. Agron. J. 81:783786

Jones, R.L. 1989. The effect of soil and foliar applied fertilizer on yield of tomato. MS Thesis. San Houston State 'Univ., Huntsville, Texas.

Landels, S.P., A. Leder, and N. Takei. 1990. Controlled release fertilizers. Chemical economics handbook. SRI International, Menlo Park, Calif. 